\title{
La mirada de las profesoras coordinadoras de la intervención pedagógica en la carrera de Pedagogía con Énfasis en I y II Ciclo
}

\author{
Coordinators' Perspective on the Pedagogical Intervention in the Study Program \\ "Pedagogy with a specialization in the $1^{\text {st }}$ to $6^{\text {th }}$ grades of the Basic General Education"
}

\author{
Maureen Camacho Oviedo ${ }^{1}$ \\ División de Educación Básica \\ Centro de Investigación y Docencia en Educación \\ Universidad Nacional \\ Heredia, Costa Rica \\ maureen.camacho.oviedo@una.cr \\ Kattia Rojas Acevedo \\ División de Educación Básica \\ Centro de Investigación y Docencia en Educación \\ Universidad Nacional \\ Heredia, Costa Rica \\ krarojasuna@gmail.com
}

Recibido 08 de abril de 2013 • Corregido 16 de agosto de 2013 • Aceptado 22 de agosto de 2013

1 Máster en Educación con Énfasis en Docencia Universitaria. Licenciada en Ciencias de la Educación con concentración en Educación Básica I y II Ciclos. Actualmente, se desempeña como Subdirectora de la División de Educación Básica (DEB) del Centro de Investigación y Docencia en Educación (CIDE) de la Universidad Nacional (UNA) y como Responsable del Programa de Desarrollo Académico y Gestión Organizacional de la División de Educación Básica. Como académica de la DEB, en la Carrera de Pedagogía con énfasis en I y ll Ciclos de la Educación General Básica, se ha desempeñado en el área de docencia, en los procesos de Intervención Pedagógica y como Responsable de la carrera. Ha laborado como docente de primaria en centros educativos públicos y privados. Posee publicaciones en el campo de la Pedagogía.

2 Máster en Pedagogía con Énfasis en Diversidad de los Procesos Educativos. Licenciada en Ciencias de la Educación con Énfasis en I y II Ciclo. Actualmente, se desempeña como Profesora de Educación General Básica en el Ministerio de Educación Pública y como académica de la División de Educación Básica (DEB) del Centro de Investigación y Docencia en Educación (CIDE) de la Universidad Nacional (UNA) en la Carrera de Pedagogía con énfasis en I y II Ciclos de la Educación General Básica. 
URL: http://www.una.ac.cr/educare

CORREO: educare@una.cr

Resumen. El presente artículo científico surge del proceso de intervención pedagógica (IP) que se realiza como parte de la formación docente en las carreras de la División de Educación Básica en la Universidad Nacional y pretende destacar las acciones del proceso de práctica profesional supervisada ejecutado en la carrera de Pedagogía con Énfasis en I y II Ciclo de la Educación General Básica, desde la visión de las académicas responsables de la coordinación de dicho proceso. Para ello, se comparte una serie de acciones de coordinación que requiere el proceso de práctica y que son fundamentales para su desarrollo y exitosa culminación. Se sistematizan en tres áreas principales con su correspondiente análisis: acciones de coordinación, acciones de inducción y acciones de cierre de la intervención pedagógica. Asimismo, se retoman las opiniones del estudiantado y profesorado de apoyo en la valoración de dichas acciones. Entre los principales aportes del artículo se destaca que el proceso de intervención pedagógica es mucho más que la formulación conjunta de un programa de curso o de instrumentos, ya que implica una serie de estrategias de coordinación entre las profesoras responsables de la intervención pedagógica, para complementar el proceso y permitir hacer de la práctica un espacio de aprovechamiento y reflexión sobre la realidad educativa. Se genera, además, el análisis y trabajo en equipo, para la constante retroalimentación del estudiantado y del mismo proceso, lo cual permite perfilar la práctica profesional renovada y fortalecer la formación universitaria, al ser el proceso de IP de la División de Educación Básica un espacio de investigación permanente.

Palabras claves. Intervención pedagógica, praxis pedagógica, coordinación, estudiantado, profesorado.

Abstract. This scientific paper is the result of the pedagogical intervention process carried out as part of teacher training in the study programs of the Basic Education Division at Universidad Nacional (Costa Rica). It aims at bringing into focus the tasks of the supervised professional practice process conducted in the study program of "Pedagogy with a specialization in the 1st to 6th grades of the Basic General Education", from the perspective of the coordinators of this process. The coordination tasks that are essential for the development and successful completion of the professional practice process are analyzed and systematized in three main areas: coordination, induction, and conclusion of the pedagogical intervention tasks. The opinions of students and faculty members are considered in the evaluation. One of the main contributions of this paper is calling attention to the pedagogical intervention process, which involves not only the joint preparation of a course program, but also a number of coordination strategies among the persons in charge of the pedagogical intervention to complete the process and take advantage of the practice to think carefully on the educational reality. This study has been also an opportunity of analysis and teamwork focused on continuous feedback to students and to the process itself, giving a new shape to the professional practice, and strengthening university training where the pedagogical intervention process of the Basic Education Division is a permanent research platform.

Keywords. Pedagogical intervention process, pedagogical praxis, students, coordination, teachers.

Para la comprensión de la temática que a continuación se expone, es necesario circunscribirse en los planteamientos de la División de Educación Básica (DEB) en cuanto a la formación académica en sus carreras de Pedagogía y, específicamente, en los procesos de intervención pedagógica (IP) en el diplomado y bachillerato. 
Las profesoras o profesores encargados del curso de Construcción Pedagógica desde el Aula Escolar, como un proceso de salida a nivel de diplomado, son quienes coordinan y organizan las acciones relativas a los procesos de Práctica Profesional Supervisada (PPS) desarrollada por el estudiantado, así como, las acciones de supervisión del profesorado destacado por la universidad para la evaluación de dichos procesos en las instituciones educativas. A partir de estos planteamientos se pretende dar a conocer las acciones de coordinación propias de los procesos de práctica del nivel de Diplomado de la carrera de Pedagogía con Énfasis en I y II Ciclo, en los grupos 22 y 32, desde la visión de las profesoras coordinadoras de la IP, durante el año 2010. Cabe mencionar que ambos grupos realizan un diplomado común, con la particularidad de que el grupo 22 está constituido por el estudiantado postulante de la carrera en Pedagogía en I y II Ciclo de la Educación General Básica, y el grupo 32 está constituido por el estudiantado postulante a Educación Especial con énfasis en Integración, a partir del bachillerato.

Se inician las acciones organizando las diferentes etapas del proceso de IP y se considera retomar los principales resultados del Informe de intervención pedagógica del año 2009 (Rojas y Segura, 2009), con el propósito de dar seguimiento y sostenibilidad a los procesos y pretendiendo una mejora sistemática. Cabe mencionar que, además de considerar dicho informe como un insumo importante para las acciones de coordinación del año 2010, se valora la experiencia de una de las académicas, quien coordinó la IP también durante los años 2008 y 2009.

El presente trabajo constituye la visión y el sentir de las profesoras responsables del proceso de IP, en diferentes momentos y ante determinadas circunstancias, plantea acciones entre las coordinadoras de la IP, el equipo de profesores y profesoras observadoras (PO) y el estudiantado.

La pertinencia de la presente indagación radica en develar cómo se articulan, en la realidad, las acciones entre las profesoras coordinadoras a nivel académico, técnico y organizacional, desde el inicio de la práctica profesional docente, hasta su cierre. Asimismo, se pretende aprender de las lecciones pasadas y orientar el proceso de práctica hacia los cambios socioeducativos e históricos, y brindar un trabajo ajustado a los planteamientos curriculares de la carrera en Pedagogía con Énfasis en I y II Ciclo de la Educación General Básica.

La práctica supervisada es entendida en la DEB como parte de los procesos de IP y constituye un aspecto relevante para las estudiantes y los estudiantes universitarios, pues es un espacio que permite que confluyan aquellos saberes y aprendizajes que han desarrollado durante su formación académica. Para ello, se plantean acciones, de manera que el estudiantado, desde el inicio hasta el cierre de su diplomado, pueda reflexionar e ir planteándose, en conjunto con sus pares y con sus profesoras y profesores, la comprensión de las diferentes realidades sociales y educativas, y la visión de los procesos cognitivos que propicia el proceso IP. 
URL: http://www.una.ac.cr/educare

CORREO: educare@una.cr

\section{Planteamiento teórico}

\section{Dinámica universitaria y organización curricular en el plan de estudio}

Para comprender la relevancia, pertinencia y coordinación del proceso de IP es importante contextualizarlo dentro de la dinámica universitaria y la organización curricular del plan de estudio, en específico en el plan de la carrera de Pedagogía con Énfasis en I y II Ciclo de Educación General Básica, ofrecida por la División de Educación Básica de la Universidad Nacional de Costa Rica (UNA).

El proceso de IP se enmarca, primeramente, en los principios del Modelo Pedagógico de la Universidad Nacional. Se mencionan los principales:

Respeto a la diversidad en todas sus expresiones; ... formación de profesionales solidarios y comprometidos con el bienestar social, flexibilidad para conceptuar el aprendizaje como proceso sociocultural, histórico, dinámico y transformable, posible y que puede construirse de muchas maneras; interacción en los procesos formativos donde los conocimientos sean discutidos y enriquecidos permanentemente, formación de un espíritu investigador en los futuros profesionales, creatividad que permita la innovación, así como la utilización de medios, estrategias y recursos de enseñanza en los procesos de mediación pedagógica, ... evaluación como proceso integral, concertado, permanente, contextualizado y propositivo ... (Universidad Nacional [UNA], 2008, p. 5)

La IP fomenta estos principios, pues promueve variedad de conocimientos conceptuales y procedimentales, así como la destreza para la investigación. Además, pretende que el estudiantado genere procesos creativos y reflexivos en el desarrollo de su práctica profesional; de manera que se deben concebir espacios evaluativos dentro del aula escolar, así como a nivel universitario, valorándose el propio desenvolvimiento como estudiante-docente, para realizar los ajustes necesarios en el proceso y fortalecer las dinámicas que han sido pertinentes y constructivas de conocimiento; siempre respetando la diversidad, considerando la formación docente adquirida y el contexto que enmarca los procesos de práctica.

Además, se propone una práctica reflexiva, que implica valorar, por parte del estudiantado, su propia experiencia de práctica, construir nuevos conceptos e impulsar propuestas de mejora, de tal manera que el proceso adquiera sentido y significado, y se convierta en un espacio de investigación permanente y una oportunidad para perfilar su futura práctica profesional. Como señala Sanjurjo (2009), el proceso reflexivo es:

Aquel que permite darnos cuenta y dar cuenta de nuestras propias acciones y de la diversidad de condicionantes que las determinan. La reflexión se constituye en un puente entre la teoría y la práctica, entre el pensamiento y la acción, entre los materiales curriculares, lo metodológico y los problemas que nos plantea la realidad. (p. 23) 
La reflexión implica valorar diversas dimensiones y supone una conciencia profesional, con el fin de mejorar lo que se hace. Esto abarca al estudiantado y al profesorado observador y coordinador de los procesos de IP.

Asimismo, el proceso de IP pretende que el estudiantado valore no solamente el contenido que va a desarrollar en las aulas escolares, sino también, cómo los va a mediar, qué conocimientos colaborará en construir con ellos, cuál es la pertinencia de las estrategias a plantear, cuándo las desarrollará, cómo integrará las temáticas al contexto. Como señalan Aebli, Colussi y Sanjurjo (1998):

En las decisiones que se toman acerca del contenido escolar, se atraviesan problemáticas científicas, ideológicas, políticas, sociales, entre otras. Pero la especificidad de esta decisión es un problema pedagógico, un problema didáctico, ya que se trata de resolver cómo se puede aprender un contenido específico, para organizar mejor su enseñanza.(...), el docente debe saber qué hacer para enseñar un contenido científico de manera que pase a ser un contenido aprendido, en un contexto determinado. (p. 47)

Según lo anterior, las decisiones sobre los contenidos por desarrollar se relacionan no solo con situaciones pedagógicas, sino también articula aspectos socioculturales. Además, las temáticas deben ser significativas, pues deben contribuir con la socialización y formación de las nuevas generaciones. El proceso educativo es parte de los elementos o aspectos en estudio durante los procesos de IP, pues se valora las relaciones entre docentes y estudiantes; las responsabilidades del estudiante-docente como eje promotor de su propia práctica, como impulsor y evaluador de esta; la evaluación como un elemento intrínseco de la práctica y que permite generar reflexión y retroalimentación al proceso.

La PPS es parte de las políticas que fueron aprobadas por la Universidad Nacional y está contemplada en su Estatuto Orgánico, en el artículo 200 inciso d, al indicar como deber de las estudiantes y los estudiantes el "cumplir con el ejercicio profesional supervisado dentro del territorio nacional, como parte de su formación universitaria y de su retribución a la sociedad costarricense, de acuerdo con lo estipulado en el reglamento que se elabore." (UNA, 1993, p. 43).

Para su cumplimiento, se ha definido el documento denominado Políticas y lineamientos para el establecimiento de la práctica profesional supervisada, el cual plantea, como parte de la responsabilidad estudiantil, que la PPS es de cumplimiento obligatorio de todos los estudiantes del nivel de bachillerato universitario o, en su defecto, de licenciatura. En el caso de la DEB, al considerar el nivel de diplomado como una salida lateral titulada del Plan de Estudio en la carrera de Pedagogía con Énfasis en I y II Ciclo de la Educación General Básica, se realiza una práctica supervisada al concluir ese nivel. Esta normativa universitaria conceptúa la PPS como: 
URL: http://www.una.ac.cr/educare

CORREO: educare@una.cr

una práctica académica estudiantil que contribuye a consolidar los conocimientos y destrezas adquiridas, así como al desarrollo de actitudes y valores. Es una experiencia que realiza el estudiante en un ámbito específico de aplicación de su especialidad disciplinaria o profesional, y a su vez, un espacio de aprendizaje que posibilita la proyección social del estudiante y el establecimiento de relaciones teóricas, metodológicas y técnicas con la realidad. (UNA, 1998, p. 1)

Como puede identificarse, para la UNA el objetivo de la PPS es consolidar los conocimientos y destrezas, así como desarrollar actitudes y valores en el estudiante-docente. Se debe convertir esta experiencia en un espacio de aprendizaje que logre la proyección social, para crear relaciones entre lo aprendido con la realidad educativa. Por ello, se establece que todos los planes de estudio deben contemplar en su diseño curricular a la PPS, conforme con las políticas y lineamientos establecidos por la universidad.

Debe señalarse que los procesos de práctica pedagógica en la DEB son organizados desde un curso en específico, en el caso de la carrera de Pedagogía con Énfasis en I y II Ciclo, el curso es Construcción Pedagógica desde el Aula Escolar y es uno de los cursos que da seguimiento a la inserción que experimenta el estudiantado durante su formación en el nivel de diplomado e intensifica este acercamiento con la realidad educativa, sus características y desafíos.

Dentro de la estructura curricular del Plan de Estudios de la carrera en Pedagogía con Énfasis en I y II Ciclos de la Educación General Básica, se define la práctica profesional supervisada como:

El proceso de práctica profesional supervisada recibe el nombre de Intervención Pedagógica en la División de Educación Básica, la misma consiste en la paulatina integración del estudiante a la cultura escolar, iniciando en el Diplomado y finalizando en el Bachillerato con proyección hasta la Licenciatura. La intervención pedagógica presupone epistemológicamente una construcción de conocimiento basada en una relación prácticateoría-práctica. Se parte del supuesto de que la intervención tiene tres momentos, uno en que el alumno observa y sistematiza el quehacer docente en un grupo escolar, un segundo momento en que el estudiante no sólo sistematiza sino que inicia ciertas labores a cargo de un grupo específico en un centro educativo, y finalmente un momento en el cual el estudiante toma el control de un grupo de niños en un centro educativo durante al menos un día laboral. (Bejarano, Flores, et al., 2010, p. 74)

Dicha definición reitera la importancia de la inserción del estudiantado en la realidad educativa y de la pertinencia de realizar la intervención pedagógica en tres momentos secuenciales, de manera que el curso de Construcción Pedagógica desde el Aula Escolar es el que organiza y coordina el tercer momento donde la estudiante o el estudiante 
universitario asume a un grupo en una institución educativa durante, al menos, una jornada escolar. Además, cabe mencionar que en las estructuras curriculares, a razón del seguimiento del eje práctico-pedagógico, los planes de estudio de las carreras de la DEB establecen un curso eje de práctica para cada nivel, el cual se convierte en el curso núcleo de la intervención pedagógica. El curso que se utiliza como eje de práctica en la carrera de Pedagogía con Énfasis en I y II Ciclo de la Educación Básica en el último bloque del segundo nivel de diplomado es, precisamente, el curso de Construcción Pedagógica desde el Aula Escolar.

Por su parte, los planes de estudio de las carreras de la División de Educación Básica pretenden lograr la formación integral del estudiantado, para ello, organizan, además, otros aspectos como las áreas disciplinarias, los ejes curriculares y los temas transversales a desarrollar en las carreras que ofrece. Es importante ubicar al curso de Construcción Pedagógica desde el Aula Escolar en el marco de estos aspectos fundamentales del plan de estudio. Respecto de las áreas disciplinarias, se ubica en el área científico-pedagógica, el área de desarrollo humano integral y el área socio-histórico cultural.

Cabe resaltar que el curso valora el acto educativo atendiendo las exigencias educativas actuales, prioritariamente el desarrollo de un pensamiento crítico y reflexivo, tanto de lo que los estudiantes y las estudiantes observan y diagnostican, como de lo que ejecutan y evalúan como docentes en práctica. Desde esta visión, se considera como parte del proceso de formación docente, la concientización del estudiantado de sus propios procesos cognitivos. Como indica Sanjurjo (2002), la metacognición debe entenderse "no solo como una reflexión acerca del conocimiento, sino como una herramienta de amplia aplicación, como una estrategia para mejorar el propio aprendizaje, como la capacidad de conocer y mejorar las propias capacidades y limitaciones" (p. 31). Ciertamente, dentro del curso se abarcan los ejes curriculares de investigación, metacognición, prácticopedagógico y humanístico.

El eje práctico pedagógico debe permear los cursos de la carrera y, por tanto, el curso de práctica, por lo que el Plan de Estudios de la carrera de Pedagogía con Énfasis en I y II Ciclo de la Educación General Básica menciona que este eje:

Implica un proceso de inserción progresiva del estudiante a la realidad del aula, contextualizada dentro de una dinámica socio-cultural e histórica, lo que permite un encuentro del estudiante con la realidad, a partir de la cual se construyen perspectivas teóricas propias, susceptibles a la confrontación y a la reconstrucción. Parte de una relación dialéctica entre práctica-teoría-práctica. . . . Es decir se busca que el estudiante parta de sus experiencias prácticas, como un medio para investigar, analizar, y generar procesos tendientes a lograr cambios en la realidad educativa. (Bejarano, Flores et al., 2010, p. 45) 
URL: http://www.una.ac.cr/educare

CORREO: educare@una.cr

El eje práctico-pedagógico está presente en la mayoría de los cursos de la carrera, pues se pretende que el estudiantado se incorpore a la experiencia docente paulatinamente, de manera que conozca el contexto de aula, el ámbito institucional y comunal, y cómo estos últimos inciden en el proceso de aprendizaje del grupo escolar y del individuo en particular. Esta inserción permite el acercamiento de la teoría con la práctica y de la práctica con los postulados teóricos que se desarrollan en la formación docente universitaria, además, genera procesos que introducen la investigación educativa desde la realidad del acto educativo.

Asimismo, el plan de estudios de la carrera señala que cualquier otro elemento específico del proceso de IP debe ser regulado de acuerdo con la normativa dictada por las autoridades competentes de la División de Educación Básica, siempre y cuando esta esté en consonancia con las disposiciones de la normativa universitaria.

\section{Nuevas disposiciones sobre los procesos de práctica}

En el 2012, los miembros del Programa de Desarrollo Académico y Gestión Organizacional de la DEB (PDAGO) reconstruyen una propuesta denominada Disposiciones generales para la intervención pedagógica (Camacho et al., 2012), el cual es un documento que orienta y norma los procesos de práctica supervisada de las carreras de la unidad académica. La propuesta surge del interés del PDAGO de fortalecer los procesos de práctica y de la valoración de los contextos actuales de la práctica docente. La propuesta es producto del análisis de documentos previos como el desarrollado por académicas y académicos de la DEB, denominado Políticas y lineamientos que orientan el desarrollo y ejecución de la intervención pedagógica (Bejarano, Dobles et al., 2008), así como de la consulta realizada a académicas y académicos relacionados actualmente con los procesos de IP de las cuatro carreras de la DEB.

El documento propuesto se presentó al Consejo Académico de Unidad, ente que lo aprobó en la sesión ordinaria n. $10-2012$, del 27 de junio del año 2012. Permite regular, a partir del II Ciclo del 2012, los procesos de IP del nivel de diplomado de las carreras de Pedagogía con Énfasis en I y II Ciclos, Pedagogía con Énfasis en Educación Preescolar y Enseñanza del Inglés para I y II Ciclos, así como el proceso de IP del nivel de bachillerato de la carrera de Educación Especial con Énfasis en Integración.

\section{Ruta metodológica para la coordinación de IP}

Para la realización de una adecuada coordinación de los procesos de IP, se deben realizar acciones para la contextualización del proceso de práctica a la realidad educativa y universitaria. Por ello, las profesoras encargadas del curso organizaron y desarrollaron diversas acciones para la ejecución y reflexión de los procesos de IP.

A continuación, se describen algunos aspectos que requieren estrategias de coordinación, imprescindibles para un coherente y eficiente proceso organizativo de la práctica docente. 


\section{Aspectos generales del curso}

Se explicitan, a continuación, algunos aspectos generales del curso Construcción Pedagógica desde el Aula Escolar y la correlación teoría-práctica que esto implica para el profesorado y el estudiantado.

El programa del curso señala, en su descripción:

Este curso ubica al estudiante en el aula de clase de I y II ciclos en un centro educativo público, con el propósito de que analice críticamente, desde una dimensión teórica-práctica, las diversas situaciones que se presentan durante el desarrollo de los procesos de enseñanza y de aprendizaje. Actúa como síntesis de su formación docente inicial para I y II ciclos de la Educación General Básica, razón por la cual tiene también una dimensión aplicativa operativa de sus conocimientos y estrategias didácticas. ... El estudiante es evaluado "in situ" por al menos un profesor de la Unidad Académica. (Camacho y Rojas, 2010, p. 1)

Durante las sesiones presenciales del curso, el estudiantado trabaja con orientaciones metodológicas tendientes a la problematización de sus experiencias como docente-estudiante, con el propósito de generar la lectura de la realidad escolar costarricense y propiciar situaciones conducentes hacia el logro de aprendizaje significativo. En cuanto a las horas prácticas en el aula escolar, se cumple durante el ciclo con una jornada laboral de un docente regular en un centro educativo público. El estudiantado realiza los trabajos prácticos del nivel durante su experiencia de práctica profesional, para integrar saberes conceptuales, procedimentales y actitudinales, con lo cual genera procesos más significativos y provechosos en su formación universitaria.

Durante el 2010, se planteó, como objetivo general del curso, favorecer la formación de educadores en I y II ciclo que: identifiquen los aportes de la pedagogía en los procesos de enseñanza y aprendizaje; sean sensibles y conscientes de sus potencialidades y de las de sus alumnos y grupos a cargo, así como conocedores de su papel como sujetos sociales, históricos y culturales. Asimismo, se plantearon diversas temáticas: la intervención pedagógica en el aula escolar, el diagnóstico situacional, el diagnóstico en el aula, la reconceptualización del planeamiento didáctico, el aula taller, las giras pedagógicas, la familia en el proceso educativo de los educandos, entre otras (Camacho y Rojas, 2010).

Dada la naturaleza teórica-práctica del curso, la evaluación planteada tiene un carácter colegiado, de manera que las actividades y experiencias desarrolladas son valoradas desde las acciones propias en las sesiones del curso, así como en las acciones y propuestas desarrolladas en el contexto educativo escolar. La distribución porcentual de esta evaluación es de $30 \%$ y de $70 \%$ respectivamente. Las actividades abarcan los siguientes rubros evaluativos: portafolio, sistematización de observaciones, propuesta de aula taller, entre otras. Como parte de la evaluación formativa y de cierre de todo el proceso se solicita al estudiantado un informe final de IP y se organiza una presentación creativa para socializar la vivencia de práctica profesional, en una sesión de clase denominada: "La práctica, una experiencia para compartir". 
URL: http://www.una.ac.cr/educare

CORREO: educare@una.cr

\section{Acciones de coordinación de la IP}

El proceso de práctica desarrollado durante el 2010 requirió de la organización e implementación de un conjunto de acciones de coordinación que se representan en la figura 1.

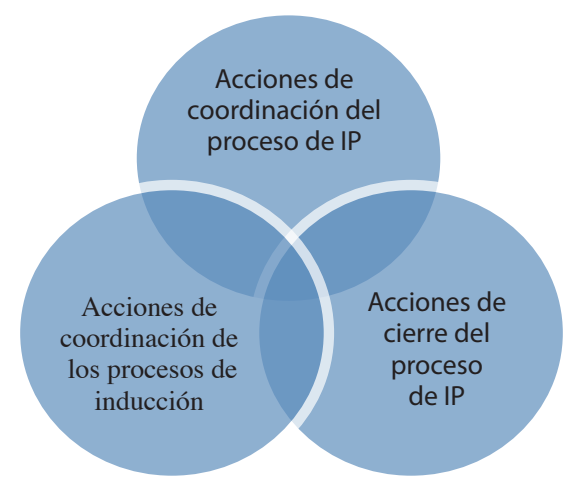

Figura 1. Acciones de coordinación del proceso de IP 2010

- Acciones de coordinación del proceso de IP. Se subdividen en acciones académicas, organizativas y administrativas:

Las acciones académicas son las referidas al proceso de mejoramiento de la IP en términos curriculares. Las acciones de organización son referidas a los aspectos técnico-pedagógicos y logísticos entre las profesoras de curso y el equipo destacado para los procesos de observación en los centros educativos. Las acciones de coordinación con el área administrativa de la DEB se refieren a la vinculación de la IP con la gestión administrativa de la unidad académica.

- Acciones de coordinación de los procesos de inducción a estudiantes, profesores observadores y centros educativos. Dichos procesos deben entenderse como las acciones tendientes a la contextualización del proceso de IP y a las funciones que cumple cada uno de los involucrados.

- Acciones de cierre de la IP. Se refieren a la reflexión y sistematización del proceso vivenciado por el profesorado y el estudiantado.

A continuación, se detallan y analizan las acciones de coordinación realizadas.

\section{Hallazgos y aciertos del proceso de coordinación}

Como se vislumbra en los apartados anteriores, el proceso de IP incluye elementos adicionales, más que la elaboración de solo un programa de curso. Requiere de estrategias coherentes y oportunas, para que el proceso de práctica sea desarrollado de la forma más 
pertinente posible, tanto en el ámbito universitario como en el escolar. El programa construido es un elemento fundamental de guía del proceso de IP, tanto para las profesoras coordinadoras, como para el estudiantado y los profesores y profesoras observadoras. Logra unificar los principios emanados del Modelo Pedagógico de la UNA, de la normativa universitaria, del plan de estudio de la carrera, así como los principales resultados del proceso de práctica anterior y las expectativas de las profesoras coordinadoras en su afán de aportar a la formación docente en el ámbito universitario.

En este apartado, se visualizan y analizan los aspectos determinantes del proceso de coordinación establecidos por las profesoras encargadas del curso de Construcción Pedagógica desde el Aula Escolar y que impactan el desarrollo y éxito del proceso de IP.

Dentro de las acciones de coordinación, se plantean varias categorías que permitirán organizar las estrategias realizadas para el desarrollo y éxito de la IP. Entre estas categorías se plantean acciones académicas, acciones organizativas, acciones propias de coordinación entre las profesoras de curso y acciones con el área administrativa de la DEB, las cuales se describen en la tabla 1.

Tabla 1

Acciones de coordinación de la IP desde el curso Construcción Pedagógica en el aula escolar, durante el II ciclo, 2010

\begin{tabular}{|c|c|}
\hline \multirow[t]{10}{*}{ Acciones académicas } & · Análisis del Informe de labores de IP 2009. \\
\hline & - Reuniones previas a la elaboración del programa de curso \\
\hline & $\begin{array}{l}\text { - Elaboración conjunta del programa del curso (selección de lecturas, instrumentos, } \\
\text { evaluación) }\end{array}$ \\
\hline & $\begin{array}{l}\text { - Implementación de cambios al programa del curso: Construcción Pedagógica } \\
\text { desde el Aula Escolar, por recomendaciones del informe de IP } 2009\end{array}$ \\
\hline & $\begin{array}{l}\text { - Elaboración conjunta de estrategias metodológicas de acción-reflexión en el } \\
\text { aula universitaria }\end{array}$ \\
\hline & $\begin{array}{l}\text { - Selección de estrategias didácticas para la reflexión de las experiencias en el aula } \\
\text { escolar }\end{array}$ \\
\hline & $\begin{array}{l}\text { - Coordinación de actividades de integración con el curso Educación para la Salud } \\
\text { y Calidad de Vida }\end{array}$ \\
\hline & $\begin{array}{l}\text { - Coordinación de una gira pedagógica en coordinación con el proyecto de Praxis } \\
\text { y gestión Social de la DEB }\end{array}$ \\
\hline & $\begin{array}{l}\text { - Organización de talleres con otras instancias de la universidad: Departamento de } \\
\text { Salud (primeros auxilios), INEINA (legislación educativa, manejo de límites) }\end{array}$ \\
\hline & $\begin{array}{l}\text { - Reuniones semanales entre las coordinadoras para registrar y analizar las } \\
\text { actividades planificadas }\end{array}$ \\
\hline
\end{tabular}

Continúa... 
URL: http://www.una.ac.cr/educare

CORREO: educare@una.cr

Acciones de organización referidas a los aspectos técnico-pedagógicos y logísticos
- Elaboración del cronograma de reuniones con PO

- Organización de un cronograma de trabajo con las actividades de IP (procesos de inducción, inicio y cierre de la IP semanas intensivas, reuniones, congresos, actividad de inducción a estudiantes y actividad de cierre)

- Planificación del proceso de inducción a PO (subdividido en dos sesiones de trabajo)

- Realización del proceso de inducción de los PO con los siguientes tópicos:

- Revisión de los principales resultados del proceso de IP 2009

- Revisión y análisis del programa de curso: Construcción Pedagógica desde el Aula Escolar, con el propósito de consensuar el proceso de evaluación de la IP y definir los criterios de evaluación con los PO

- Revisión y análisis del documento: Políticas que norman el Proceso de Práctica de las cuatro carreras de la División de Educación Básica

- Revisión y análisis de la Guía para la observación de la IP

- Elaboración de un instrumento para la evaluación de las actividades definidas como extracurriculares

- Distribución de estudiantes considerando los siguientes criterios: ubicación, disponibilidad horaria de PO, cantidad y zona escolar

- Entrega a cada profesor de un expediente completo por cada estudiante, con los siguientes documentos: ficha personal, ficha institucional, guías para las observaciones, instrumentos para reuniones con los estudiantes y otros documentos)

- Respuesta oportuna a las consultas o dudas hechas por los PO y estudiantado

- Asignación de ocho estudiantes por profesor(a) observador(a) nombrado(a) por un tiempo de 10 horas semanales

- Elaboración de un banco de datos del proceso de IP (cronogramas, agendas, instrumentos, instrumentos, consultas, entre otros)

- Habilitación de un correo grupal como canal de comunicación con el equipo de $\mathrm{PO}$, para enviar información adicional

- Realización de reuniones una vez al mes u organización de reuniones por situaciones emergentes (ocho reuniones contando con agenda, control de asistencia, recursos materiales, otros)

- Mediar situaciones en situaciones conflictivas entre el PO y el estudiante

- Planificación y elaboración conjunta de actividades e instrumentos de evaluación

- Aplicación del instrumento de cierre de la IP a PO al estudiantado, vía digital

- Organización de la actividad de cierre de la IP del estudiantado

Continúa... 


\begin{abstract}
Acciones de coordinación con la gestión administrativa

- Realización de entrevistas a los postulantes al cargo de PO

- Elaboración de un cuadro con información personal y horaria de los postulantes al puesto de PO y envío a Dirección de la DEB

- Gestión de cartas y carné de la universidad para los PO, que los acredite como profesores de la Universidad Nacional

- Comunicación escrita las fechas de las semanas intensivas a los académicos y académicas del nivel

- Gestión inicial de pago de viáticos para los PO

- Entrega de cartas para los centros educativos anfitriones con el listado de estudiantes ubicados en ese centro educativo

- Gestión de cartas para cada estudiante-practicante, para acreditarlos como estudiantes de la Universidad Nacional

- Gestión de la póliza de seguro de cada estudiante para que la entreguen en cada centro educativo.

- Elaboración de cartas para cada docente anfitrión con información personal y funciones y responsabilidades del estudiante durante el proceso de IP

- Solicitud ante la oficina administrativa del CIDE el auditorio para diversas actividades: proceso de inducción del estudiantado, talleres, cierre del proceso, otros
\end{abstract}

De la tabla 1 se desprende que las docentes encargadas del curso Construcción Pedagógica desde el Aula Escolar asumen la responsabilidad de organizar y ejecutar acciones de coordinación, desde el inicio del proceso hasta su cierre. Estas acciones se subdividen en técnicas pedagógicas que requieren de un proceso sistemático de coordinación, que inicia con la valoración del informe de IP del año anterior, la categorización de procesos y la definición de las acciones y estrategias por ejecutarse durante todo el proceso. Posteriormente, se propicia la integración de aprendizajes con acciones conjuntas entre algunos académicos o académicas del nivel, con el propósito de realizar esfuerzos para realizar integraciones curriculares; así como con el equipo de profesores observadores, otras unidades académicas, proyectos de la DEB y con la gestión administrativa, tanto para el desarrollo del curso como del proceso general de IP.

Se refleja que las docentes encargadas del curso coordinan la gran mayoría de las actividades en conjunto: incorporación de recursos universitarios, interdivisiones y departamentales. Asimismo, la coordinación asume la capacitación y seguimiento total del equipo de PO, debido a que hasta el momento no se da la consolidación de un equipo de profesores observadores de la DEB, aunado esto con situaciones imprevistas que se presentan, como la renuncia de dos profesoras observadoras al inicio del proceso por situaciones personales, situación que generó atrasos para el comienzo de la práctica de algunos estudiantes. Así como los cambios en la planificación de actividades programadas por uno de los PO y su no disposición para seguir los acuerdos tomados con el resto del equipo, aspecto que generó incertidumbre y confusión entre el estudiantado y otros de los profesores observadores. 
URL: http://www.una.ac.cr/educare

CORREO: educare@una.cr

Otra de las categorías de acción propias de la coordinación fue la organización de los procesos de inducción, en la tabla 2 se plantean las acciones desarrolladas por las profesoras coordinadoras para abordar dichos procesos.

Tabla 2

Acciones desarrolladas por las coordinadoras de la IP referidas a los tres procesos de inducción, durante el II ciclo, 2010

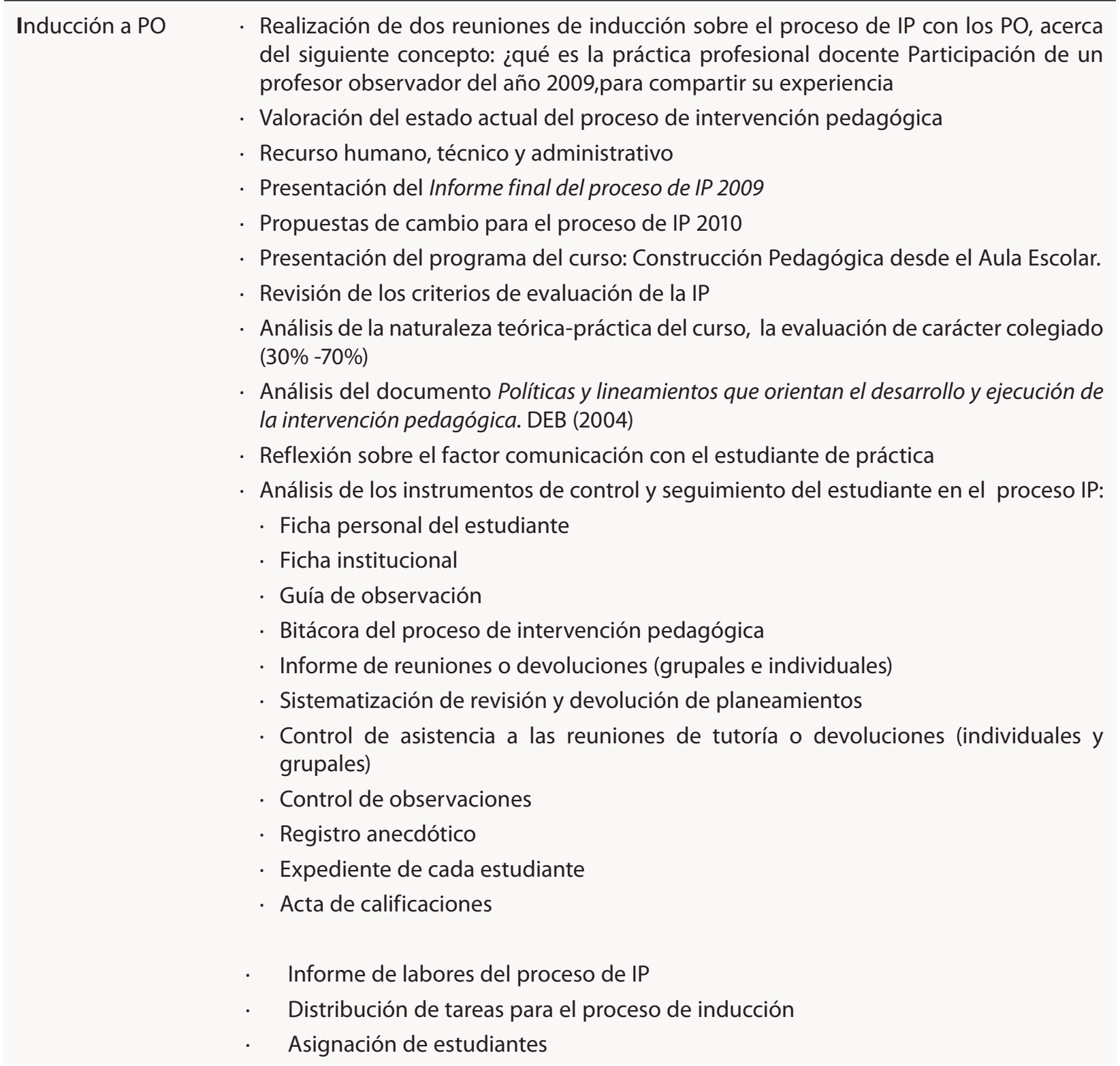




\begin{tabular}{|c|c|}
\hline $\begin{array}{l}\text { Inducción a } \\
\text { estudiantes }\end{array}$ & $\begin{array}{l}\text { - Organización del proceso de inducción a estudiantes } \\
\text { - Valoración de los aspectos importante a considerar en el proceso de inducción de IP } \\
\text { - Eistribución y planificación entre los PO de las siguientes funciones: } \\
\text { - Elaboración de una agenda de trabajo } \\
\text { - Elaboración de un desplegable con la información más destacada del proceso } \\
\text { - Programación de la actividad con los siguientes aspectos: } \\
\text { - Bienvenida } \\
\text { - Presentación del equipo de PO } \\
\text { - Motivación a cargo de un PO } \\
\text { - Presentación de la propuesta de IP para el estudiantado } \\
\text { - Análisis del documento denominado: Políticas y lineamientos que orientan el desarrollo } \\
\text { y ejecución de la intervención pedagógica } \\
\text { - Presentación de la propuesta evaluativa del proceso de IP } \\
\text { - Presentación de los aspectos mínimos que debe contener el planeamiento didáctico } \\
\text { - Espacio de consultas o dudas } \\
\text { - Distribución del estudiantado por profesor observador } \\
\text { - Refrigerio }\end{array}$ \\
\hline \multirow[t]{5}{*}{$\begin{array}{l}\text { Inducción en } \\
\text { centros educativos }\end{array}$} & $\begin{array}{l}\text { Calendarización para que los PO se presenten en cada una de las instituciones } \\
\text { educativas donde le corresponde realizar observaciones }\end{array}$ \\
\hline & $\begin{array}{l}\text { Coordinación con directores o directoras de cada centro educativo de la fecha de } \\
\text { realización del proceso de inducción, para orientar e informar sobre el proceso en } \\
\text { términos pedagógicos y técnicos-administrativos }\end{array}$ \\
\hline & $\begin{array}{l}\text { Organización del proceso de inducción en los diferentes centros educativos junto } \\
\text { con los estudiantes ubicados en cada centro educativo }\end{array}$ \\
\hline & $\begin{array}{l}\text { Elaboración de un desplegable con la información del proceso de IP y datos de las } \\
\text { académicas encargadas del proceso. }\end{array}$ \\
\hline & $\begin{array}{l}\text { Entrega del desplegable a cada docente con la información referida a los deberes y } \\
\text { derechos del estudiante y la función de ellos y ellas como docentes anfitriones }\end{array}$ \\
\hline
\end{tabular}

Se desprende de la tabla 2 que las docentes encargadas del curso Construcción Pedagógica desde el Aula Escolar, asumen la responsabilidad de organizar las acciones de cada uno de los procesos de inducción. En relación con el proceso de inducción a PO, las encargadas de coordinar la IP se ocupan de su ejecución; en relación con los procesos de inducción del estudiantado, las acciones de las coordinadoras son tendientes a la organización inicial, pero posteriormente toman la responsabilidad compartida del proceso con los PO y, por último, el proceso de inducción en los centros educativos es organizado por la coordinación de la IP junto con el equipo de PO, quienes a su vez se organizan para ejecutar el proceso de inducción con el director o directora, docentes anfitriones y estudiantado practicante ubicados en el centro educativo. 
URL: http://www.una.ac.cr/educare

CORREO: educare@una.cr

En relación con lo anterior, cabe mencionar que el cien por ciento de los $\mathrm{PO}$ y el estudiantado practicante participaron en el proceso de inducción a estudiantes. Sin embargo, la inducción en las instituciones educativas no se realizó en su totalidad, pues en algunos casos solo lo ejecutó el PO, otras solo el estudiantado practicante y otras inducciones no contaron con la participación del director o docentes anfitriones.

La inducción en los centros educativos es valorada, tanto para las coordinadoras de la IP, como para el estudiantado, como necesaria para el proceso, en razón de experiencias importantes de aprendizaje. Sin embargo, los factores de tipo administrativo de las instituciones y el tiempo máximo disponible (cuarenta minutos) por los directores o directoras, para realizar la actividad, limitan muchas veces el periodo de consultas o dudas que tienen los docentes anfitriones. Es importante reiterar que la inasistencia de algunos docentes anfitriones sigue limitando la efectividad del proceso.

La última de las acciones de coordinación se refiere al proceso de cierre de la IP, dichas acciones se presentan en la tabla 3, que incluye las estrategias desarrolladas por los diversos involucrados en el proceso de práctica supervisada.

Tabla 3

Acciones desarrolladas por las coordinadoras, los profesores y profesoras observadoras y estudiantes, referidas al proceso de cierre del proceso de la IP, 2010

\begin{tabular}{|c|c|}
\hline $\begin{array}{l}\text { Coordinadoras } \\
\text { de la IP }\end{array}$ & $\begin{array}{l}\text { Elaboración de un instrumento de evaluación de la IP, para el estudiantado y PO, el cual se } \\
\text { habilitó en línea } \\
\text { Organización de una actividad de cierre en el auditorio del CIDE, para que el estudiantado } \\
\text { socialice a nivel emotivo y como experiencia de aprendizaje lo vivido en el proceso de IP } \\
\text { Organización de una actividad de cierre para la gira pedagógica realizada con el proyecto } \\
\text { de la DEB } \\
\text { Participación en la actividad de la Feria de Salud de la DEB, organizada por la profesora } \\
\text { del curso de Salud y Calidad de Vida } \\
\text { Recepción de actas de evaluación de la IP y expedientes } \\
\text { Sistematización de la información obtenida durante el proceso por parte de las profesoras } \\
\text { coordinadoras de la IP, los PO y el estudiantado } \\
\text { Elaboración de un informe final del proceso de IP } 2010 \text { para entregarlo a la DEB }\end{array}$ \\
\hline Equipo de PO & $\begin{array}{l}\text { Entrega de promedios al estudiantado asignado y su correspondiente firma del acta de } \\
\text { evaluación } \\
\text { Entrega a las coordinadoras de la IP de las actas de evaluación correspondientes al 70\% de } \\
\text { la práctica profesional supervisada y expedientes debidamente completos } \\
\text { Entrega del informe final de IP suministrado por las coordinadoras }\end{array}$ \\
\hline Estudiantado & $\begin{array}{l}\text { Participación en el proceso de cierre de IP } \\
\text { Preparación de la presentación subgrupal para la actividad de cierre según su vivencia de IP } \\
\text { Entrega del informe final de IP suministrado por las profesoras de curso }\end{array}$ \\
\hline
\end{tabular}


Como se extrae de los datos de la tabla 3, las profesoras encargadas del curso Construcción Pedagógica desde el Aula Escolar asumen la responsabilidad de organizar las acciones de cierre del proceso de práctica supervisada, por lo cual resulta fundamental recopilar los datos y percepciones del equipo de IP y del estudiantado, para poder sistematizarlo y analizarlo posteriormente, de allí la solicitud de informes de cierre, lo que se asociará con la valoración propia de las profesoras coordinadoras.

Queda clara, también, la importancia de coordinar la evaluación del estudiantado durante el todo el ciclo lectivo, pues al final los PO entregan la evaluación realizada a sus estudiantes asignados, que se pondera con la reportada por las profesoras de curso, con el propósito de que esta calificación dé una visión evaluativa integral del proceso llevado a cabo por el estudiantado durante su práctica supervisada, sea a nivel teórico, práctico o reflexivo.

Este proceso de evaluación y reflexión incluye, como parte del cierre del proceso de práctica, la entrega de un informe por parte del equipo de PO y la sistematización de los instrumentos aplicados al estudiantado. Dicha documentación refleja aspectos muy valiosos de destacar.

En cuanto a la valoración final del proceso de inducción realizado al estudiantado, se destacan en la tabla 4, las principales apreciaciones de los estudiantes y las estudiantes de práctica.

Tabla 4

Sistematización de las apreciaciones de los estudiantes y las estudiantes en práctica supervisada, ante aspectos propios del proceso de inducción, 2010

\begin{tabular}{|c|c|c|}
\hline Categorías & Apreciaciones & Frecuencia \\
\hline $\begin{array}{l}\text { 1. Organización del proceso de } \\
\text { inducción }\end{array}$ & $\begin{array}{l}\text { - Adecuada organización y orden durante el proceso } \\
\text { - Proceso dinámico y completo } \\
\text { - Información clara y explícita } \\
\text { - Buena en general } \\
\text { - Compromiso del personal } \\
\text { - Otras }\end{array}$ & $\begin{array}{l}13 \\
7 \\
5 \\
1 \\
1 \\
1\end{array}$ \\
\hline $\begin{array}{l}\text { 2. Presentación del documento } \\
\text { sobre las Políticas que orientan } \\
\text { el proceso de la intervención } \\
\text { pedagógica }\end{array}$ & $\begin{array}{l}\text { - Presentación suficientemente clara } \\
\text { - Se aclararon dudas } \\
\text { - Explicación breve y rápida } \\
\text { - } \text { - Muló más preparación porganizada } \\
\text { - Presentación de los temas más importantes de la práctica } \\
\text { - Entrega de material en físico a los estudiantes para la } \\
\text { presentación de la información oral }\end{array}$ & $\begin{array}{l}13 \\
3 \\
3 \\
2 \\
1 \\
1 \\
1\end{array}$ \\
\hline
\end{tabular}

Continúa... 
URL: http://www.una.ac.cr/educare

CORREO: educare@una.cr

\begin{tabular}{|c|c|c|}
\hline Categorías & Apreciaciones & Frecuencia \\
\hline $\begin{array}{l}\text { 3. El proceso de inducción: } \\
\text { dudas de su rol en la } \\
\text { intervención pedagógica } \\
\text { y las funciones del } \\
\text { profesorado observador }\end{array}$ & $\begin{array}{l}\text { - El proceso me permitió aclarar dudas } \\
\text { - Aclara el trabajo a realizar en cuanto a responsabilidades y } \\
\text { - No fueron aclaradas todas las dudas } \\
\text { - Permitió una visión más general del proceso } \\
\text { - Espacio para preguntas fue importante } \\
\text { - Mucha información para un solo momento, debe ser más puntual } \\
\text { - Aclaración de dudas permite seguridad } \\
\text { - Se reforzó lo ya aclarado por la profesora de Construcción } \\
\text { Pedagógica }\end{array}$ & $\begin{array}{l}3 \\
2 \\
2 \\
2 \\
1 \\
1\end{array}$ \\
\hline $\begin{array}{l}\text { 4. Dudas que persisten después } \\
\text { del proceso de inducción }\end{array}$ & $\begin{array}{l}\text { - Las dudas fueron aclaradas } \\
\text { - Algunas que se considera se podrán resolver con el profesor } \\
\text { (a) observador(a) o profesoras de curso } \\
\text { - Específicas al trabajo de aula (planeamiento, disciplina, } \\
\text { problemas de los niños, uso de material, adecuaciones, uso de } \\
\text { libros de texto) } \\
\text { - Ninguna } \\
\text { - Dudas que pueden surgir durante el proceso de práctica } \\
\text { - Específicas a documentos (función del registro) } \\
\text { - Específicas a situaciones inesperadas (no hay lecciones) }\end{array}$ & $\begin{array}{l}2 \\
1 \\
1 \\
1\end{array}$ \\
\hline $\begin{array}{l}\text { 5. Valoración del proceso } \\
\text { de inducción en términos } \\
\text { generales }\end{array}$ & $\begin{array}{l}\text { - Proceso muy importante que permite aclarar muchas dudas y } \\
\text { - Bueno } \\
\text { - Proceso que permite tener una visión más clara y cercana de la } \\
\text { práctica } \\
\text { - Proceso claro y específico } \\
\text { - Provechoso y adecuado } \\
\text { - Completo (se abarca todos los temas) } \\
\text { - Excelente } \\
\text { - Muy bueno } \\
\text { - Organizado con el esfuerzo de las profesoras y profesores } \\
\text { - Prepara al alumno y le da seguridad } \\
\text { - Permite conocer a los profesores observadores }\end{array}$ & $\begin{array}{l}5 \\
5 \\
4 \\
3 \\
2 \\
2 \\
1 \\
1 \\
1 \\
1 \\
1 \\
1\end{array}$ \\
\hline $\begin{array}{l}\text { 6. Sugerencias o } \\
\text { recomendaciones }\end{array}$ & $\begin{array}{l}\text { - Proceso muy bueno y ameno } \\
\text { - Ninguna } \\
\text { - Manejo del tiempo } \\
\text { - Mayor orden y manejo de la voz por parte de los expositores } \\
\text { - Aporte al proceso, ubica al estudiante y lo introduce al proceso } \\
\text { de práctica } \\
\text { - Proceso bien organizado } \\
\text { - Revisión del sonido } \\
\text { - Brindar en físico los temas tratados } \\
\text { - Ser más específicos en ciertos puntos } \\
\text { - No limitar la IP al Circuito } 02 \\
\text { - Enviar presentaciones a la dirección de correo de los estudiantes }\end{array}$ & $\begin{array}{l}1 \\
1 \\
1 \\
1 \\
1 \\
1\end{array}$ \\
\hline
\end{tabular}


Al referirse el estudiantado a su proceso de inducción, se observa una reiteración en las apreciaciones de que este proceso les fue muy útil para aclarar dudas existentes, así como para conocer los lineamientos que norman la IP y las responsabilidades de cada uno de los involucrados en el proceso de IP, entiéndase profesoras coordinadoras, profesoras y profesores observadores, docentes anfitriones y anfitrionas y estudiantes en práctica supervisada.

La descripción del proceso de IP es fundamental, pues le permite al estudiantado tener una visión general del proceso de práctica. Se destaca, también, que el momento de conocer a las profesoras y profesores observadores (PO) correspondientes a cada estudiante es un espacio emotivo y vital para establecer un primer contacto, en donde se intercambian diferentes puntos de vista y se establecen ciertas disposiciones, según la perspectiva de cada profesor o profesora, para desarrollar la IP. Además, este espacio le permite, al estudiantado, comprender que las dudas que surjan durante el proceso o consultas específicas de la IP pueden resolverlas con su PO. Como se deduce, tanto la inducción como el acercamiento con los PO, genera más confianza y seguridad en el estudiantado practicante.

Además, se recalca en las apreciaciones obtenidas que el proceso de inducción fue propiciado de forma adecuada. Se destaca la labor de organización, que el proceso fue claro, se desarrollaron muy bien los aspectos referentes a la IP, se comprendieron los lineamientos que norman la práctica en la DEB y, en su gran mayoría, las dudas fueron evacuadas, generando un espacio de información útil y provechosa para el estudiantado.

Entre las opciones de mejora, planteadas por el estudiantado en la tabla 4, se señalan asuntos puntuales que pueden ser atendidos en próximos momentos de práctica, como son los aspectos meramente técnicos de sonido y el aporte de material en físico para el estudiantado.

Es valioso destacar que el proceso de inducción a los docentes y las docentes anfitrionas es una actividad más de coordinación de la IP y es una actividad esencial para iniciar la IP de forma adecuada. Según la experiencia acumulada de los $\mathrm{PO}$, se recomienda que los procesos de inducción en centros educativos debe realizarse antes de que el estudiantado inicie formalmente sus visitas o aplicaciones en dichas instituciones. Esto, debido a que el PO debe cerciorarse que los docentes y el administrador o administradora del centro educativo estén realmente interesados en participar del proceso y reflexionar sobre si cumplen con el perfil esperado para los procesos de práctica supervisada de la DEB. Este proceso es importante como puente de comunicación entre los actores del proceso y, a su vez, como una actividad que promueve un acercamiento de las instituciones educativas con la Universidad Nacional, específicamente la DEB como instancia formadora de docentes, lo cual se ejemplifica en una de las opiniones de los PO: "Se establecieron importantes nexos entre los actores principales del proceso: docentes, profesores observadores y estudiantes practicantes".

Para efectos de organización, los PO se distribuyen entre las instituciones educativas anfitrionas y tienen la función de coordinar con los directores o directoras de las escuelas el tiempo para realizar la actividad. Se recomienda ejecutarla con la presencia del director o directora del centro educativo, los educadores que fungirán como docentes anfitriones o 
URL: http://www.una.ac.cr/educare

CORREO: educare@una.cr

anfitrionas, así como con los estudiantes y las estudiantes practicantes, de manera que cada estudiante obtenga un seguimiento más integral de su proceso de práctica.

Esta inducción, además, permite aclarar dudas al profesorado, como se destaca en la opinión de uno de los PO: "Existían muchas expectativas por parte de docentes de la institución, gran variedad de inquietudes acerca del proceso. Es primordial que la inducción se realice, y se caracteriza como un proceso: "Enriquecedor y valioso ya que se toman elementos esenciales para el proceso". Se resalta el papel del PO en dicho proceso y el uso de estrategias para lograr quelas inducciones sean exitosas, a pesar de algunos obstáculos que se presentan, entre ellos: el poco tiempo disponible para el tratamiento de todas temáticas de inducción, la ausencia de algunas o algunos docentes y la ausencia en gran parte de los directores y las directoras, como se denota en las frases textuales de dos de los PO: "Existen limitantes de tiempo para el tratamiento correcto de cada una de las temáticas sobre todo en el área de la didáctica", y "Debido a que el proceso de la IP es bastante complejo existió un faltante de tiempo para abarcar con tranquilidad cada una de las áreas".

Para finalizar con los elementos y aciertos del proceso de IP, se presentan a continuación una serie de opiniones de los PO sobre todo el proceso de coordinación desarrollado por las profesoras encargadas de curso y su responsabilidad como coordinadoras de la IP del año 2010.

Se puede evidenciar que, en su mayoría, los PO coinciden en que el trabajo de coordinación de las profesoras responsables del proceso de IP fomentó la unidad y el trabajo en equipo por parte de las profesoras coordinadoras, quienes funcionaron como mediadoras y orientadoras del proceso de IP. Lo cual se refleja en las siguientes frases: "Es muy importante la forma en la que las profesoras coordinadoras trataron de fomentar la unidad y el trabajo en equipo, como parte importante del adecuado desarrollo de la IP", "La comunicación fue manejada de manera asertiva por parte de las profesoras coordinadoras". Además, destacan que las reuniones permitieron no solo organizar las acciones, sino que hicieron que el proceso fuera más enriquecedor, que la opinión de otros compañeros o compañeras ayudara a la propia experiencia como profesor o profesora observadora, lo que se evidencia en las siguientes opiniones: "El proceso de inducción, las reuniones mensuales y la atención a los caso especiales siempre se dio en el clima adecuado para el buen desarrollo de la IP", "Se realizaron las reuniones necesarias según lo establecía el cronograma. Las reuniones se desarrollaron en un clima de respeto, interacción adecuada con las coordinadoras del proceso y lo más importante los aportes innumerables que todos los docentes brindaron al proceso", "Las reuniones realizadas sirvieron para evacuar dudas en forma general".

Se menciona que hubo un clima de respeto y una visión de trabajo en equipo, lo cual es fundamental en estos procesos; sin embargo, también se anota que la falta de experiencia o la falta de "humildad" por parte de algunos PO entorpece el trabajo o puede dificultarlo, lo que se expresa en la siguiente opinión: "Sin embargo existen personas que les falta experiencia y humildad, lo que entorpece la labor. Es recomendable un proceso de concientización más profundo hacia los nuevos docentes observadores que participen en la IP". 
Asimismo, las inducciones realizadas por parte de las profesoras coordinadoras a los profesores o profesoras observadoras de reciente ingreso, ayudan a los mismos a incorporarse mejor en el proceso, lo que se denota en los siguientes comentarios: "La inducción que las profesoras coordinadoras realizaron a las profesoras observadoras y a los estudiantes practicantes marcaron la pauta para el desarrollo adecuado de la IP. El uso de los recursos tecnológicos fue fundamental para mantener una constante comunicación entre los participantes del proceso", "La inducción y las reuniones mensuales permiten incorporarse de forma exitosa al proceso de IP", "Se llevó a cabo de forma satisfactoria, siguiendo los lineamientos que orientan el desarrollo y ejecución de la IP". Cabe destacar, entonces, que el asesoramiento a los PO que se están incorporando al proceso debe ser un elemento constante y que se requiere de cierto tiempo para que el profesor observador o profesora observadora se familiarice por completo con el proceso de IP y logre darle un seguimiento adecuado y atinente al estudiantado.

\section{Reflexiones finales y retos}

En relación con el proceso de práctica desarrollado durante el 2010, se puede decir que la organización e implementación de un conjunto de acciones de coordinación fueron fundamentales para el éxito de la intervención pedagógica, por lo que se destacan a continuación, algunas reflexiones y retos importantes:

- El proceso de práctica profesional supervisada de la División de Educación Básica genera un espacio de investigación permanente para el estudiantado, que le permite realizar cambios en el proceso y perfilar su futura práctica profesional.

- El planteamiento del curso de Construcción Pedagógica desde el Aula Escolar, que respalda el proceso de IP, posee coherencia curricular, pues cumple con las políticas establecidas por la UNA para la práctica supervisada, con lo planteado en el Plan de Estudio de la Carrera en Pedagogía en I y II Ciclo, con lo estipulado por el Modelo Pedagógico y con la normativa universitaria, fortalecido el proceso por la propuesta metodológica y evaluativa diseñada por las profesoras asignadas al curso.

- El proceso de práctica profesional supervisada logra insertar al estudiantado en la realidad educativa del aula costarricense.

- La IP permite promover la formación de educadoras y educadores conocedores de su papel como sujetos sociales, históricos y culturales.

- Una característica de la organización del proceso de práctica es su flexibilidad, lo que posibilita, adecuadamente, al estudiantado el desarrollo de propuestas pedagógicas.

- El trabajo en equipo del profesorado y el estudiantado es esencial para darle al proceso de IP coherencia y unidad en todo el esfuerzo universitario. 
URL: http://www.una.ac.cr/educare

CORREO: educare@una.cr

- El espacio y tiempo asignado es fundamental para la coordinación eficaz entre las profesoras encargadas de los cursos responsables del proceso de práctica supervisada.

- El proceso de inducción es un espacio para que el estudiantado conozca desde un inicio las acciones académicas y evaluativas a desarrollar durante el proceso de IP.

- El estudiantado califica el proceso de inducción como un espacio relevante, esclarecedor de dudas y adecuadamente organizado. Sin embargo, el profesorado encargado de este proceso tiene como reto plantearlo en al menos dos etapas.

- La ejecución de la inducción en los centros educativos es primordial para el proceso de IP, pero surge el reto de programarla en sesiones de trabajo continuas, que brinden como producto final la seguridad de una institución informada, interesada e innovadora en los procesos de formación y práctica profesional docente.

- Los espacios de cierre del proceso de IP permitieron la socialización del aprendizaje por parte del estudiantado y un espacio de análisis para el profesorado.

- Con relación con los retos, existe la necesidad de consolidar un equipo de profesores observadores en la DEB, ya que los procesos de mejora a partir de las experiencias anteriores se ven afectados por la renovación constante del conjunto de profesores observadores.

- Debe considerarse el asignar un mayor tiempo para los académicos o las académicas responsables de coordinar la práctica profesional supervisada de la DEB a nivel de diplomado, pues las acciones de coordinación son amplias y requieren de mucha logística y atención de situaciones y actividades durante el ciclo.

\section{Referencias}

Aebli, H., Colussi, G. y Sanjurjo, L. (1998). Fundamentos psicológicos de una didáctica operativa. El aprendizaje significativo y la enseñanza de los contenidos escolares. Rosario: Homo Sapiens.

Bejarano, S., Dobles, C., León, H, Quesada, E., Rojas, D. y Vásquez, E. (2008). Políticas y lineamientos que orientan el desarrollo y ejecución de la intervención pedagógica desde los cursos. Heredia, Costa Rica: División de Educación Básica. Material inédito.

Bejarano, S., Flores, L., Herrera, A., Jiménez, R. y León, H. (2010). Plan de estudios de la carrera de licenciatura de pedagogía en I y II ciclos de la educación general básica. Heredia, Costa Rica: División de Educación Básica.Material inédito.

Camacho, M., Castillo, R., León, H., Miranda, A., Pereira, Z. y Vásquez, E. (2012). Disposiciones generales para la intervención pedagógica. Heredia, Costa Rica: División de Educación Básica. Material inédito. 
Camacho, M. y Rojas, K. (2010). Programa del curso DB-1212 Construcción pedagógica desde el aula escolar. (Diplomado en Pedagogía con énfasis en I y II ciclo de la Educación General Básica). Universidad Nacional. Centro de Investigación y Docencia en Educación. División de Educación Básica. Heredia, Costa Rica.

Rojas, K. y Segura, S. (2009). Informe de la Intervención pedagógica 2009. Heredia, Costa Rica: División de Educación Básica. Manuscrito inédito.

Universidad Nacional (UNA). (2008). Modelo pedagógico de la Universidad Nacional. Heredia, Costa Rica. Recuperado de http://unaweb.una.ac.cr/index.php?option=com remository\&|temid $=0$ \&func $=$ startdown\&id=141

Universidad Nacional. Consejo Universitario (1998). Políticas y lineamientos para el establecimiento de la práctica profesional supervisada. Heredia, Costa Rica. Recuperado de http://www. juridica.una.ac.cr/index.php?option=com remository\&/temid=0\&func=startdown\&id=57

Universidad Nacional. (1993). Estatuto orgánico de la Universidad Nacional. Heredia, Costa Rica. Recuperado de http://www.juridica.una.ac.cr/index.php?option=com remository\&ltemid $=0 \&$ func $=$ startdown\&id $=144$

Sanjurjo, L. O. (2002). La formación práctica de los docentes. Reflexión y acción en el aula. Rosario: Homo Sapiens.

Sanjurjo, L. O. (Coord.). (2009). Los dispositivos para la formación en las prácticas profesionales. Rosario: Homo Sapiens.

Cómo citar este artículo en APA:

Camacho, M. y Rojas, K. (2013). La mirada de las profesoras coordinadoras de la intervención pedagógica en la carrera de Pedagogía con Énfasis en I y II Ciclo. Revista Electrónica Educare, 17(3), 315-337. Recuperado de http://www.revistas.una.ac.cr/index.php/EDUCARE/issue/current

Nota: Para citar este artículo en otros sistemas puede consultar el hipervínculo "Como citar el artículo" en la barra derecha de nuestro sitio web:

http://www.revistas.una.ac.cr/index.php/EDUCARE/index 